\title{
Improved quantitative microbiome profiling for environmental antibiotic resistance surveillance
}

\author{
Amelie Ott ${ }^{1}$, Marcos Quintela-Baluja' ${ }^{1}$, Andrew M. Zealand ${ }^{1}$, Greg O'Donnell ${ }^{1}$, Mohd Ridza Mohd Haniffah² and \\ David W. Graham ${ }^{1 *}$ (1)
}

\begin{abstract}
Background: Understanding environmental microbiomes and antibiotic resistance (AR) is hindered by over reliance on relative abundance data from next-generation sequencing. Relative data limits our ability to quantify changes in microbiomes and resistomes over space and time because sequencing depth is not considered and makes data less suitable for Quantitative Microbial Risk Assessments (QMRA), critical in quantifying environmental AR exposure and transmission risks.
\end{abstract}

Results: Here we combine quantitative microbiome profiling (QMP; parallelization of amplicon sequencing and 165 rRNA qPCR to estimate cell counts) and absolute resistome profiling (based on high-throughput qPCR) to quantify AR along an anthropogenically impacted river. We show QMP overcomes biases caused by relative taxa abundance data and show the benefits of using unified Hill number diversities to describe environmental microbial communities. Our approach overcomes weaknesses in previous methods and shows Hill numbers are better for QMP in diversity characterisation.

Conclusions: Methods here can be adapted for any microbiome and resistome research question, but especially providing more quantitative data for QMRA and other environmental applications.

Keywords: Quantitative microbiome, Hill numbers, Antibiotic resistance, QMRA, River water, Southeast Asia

\section{Background}

Antibiotic resistance (AR) represents a global threat [1]. Between 2014 and 2016, more than one million people died due to drug resistant pathogen infections and increasing death tolls are expected in the future [2]. AR pathogens not only spread through hospitals, but also enter the environment via insufficiently treated sewage $[3,4]$. This is especially a problem in emerging countries. Increased economic wealth permits greater access to antibiotics while waste management often lags behind

*Correspondence: david.graham@newcastle.ac.uk

${ }^{1}$ School of Engineering, Newcastle University, Cassie Building, Newcastle upon Tyne NE1 7RU, UK

Full list of author information is available at the end of the article
[5]. However, quantifying the extent of environmental AR over space and time is difficult because methods are not standardized, with researchers using different measures of AR (e.g. antibiotics, antibiotic resistant genes, ARGs; antibiotic resistant bacteria, ARBs; and mobile genetic elements, MGEs) across studies [6]. Ideally, bacterial hosts of ARGs should be tracked [7], but reliable molecular methods that couple bacteria species and ARG abundances (e.g. epicPCR [8], Hi-C [9]) are still in their infancy. Further, linking microbiome characteristics from DNA sequencing with quantitative ARG data is an unfulfilled aspiration for studying environmental AR [10, 11]. This restricts our ability to perform realistic Quantitative Microbial Risk Assessments (QMRA) needed to quantify true risks of environment AR exposures [12, 13]. original author(s) and the source, provide a link to the Creative Commons licence, and indicate if changes were made. The images or other third party material in this article are included in the article's Creative Commons licence, unless indicated otherwise in a credit line to the material. If material is not included in the article's Creative Commons licence and your intended use is not permitted by statutory regulation or exceeds the permitted use, you will need to obtain permission directly from the copyright holder. To view a copy of this licence, visit http://creativecommons.org/licenses/by/4.0/. The Creative Commons Public Domain Dedication waiver (http://creativeco mmons.org/publicdomain/zero/1.0/) applies to the data made available in this article, unless otherwise stated in a credit line to the data. 
Correlation-based methods can develop hypotheses to guide future experimental work but they are restricted due to technical biases introduced from DNA sequencing $[7,14,15]$.

Next-generation sequencing (NGS) data are inherently compositional, providing relative abundance information at best [16]. It is impossible to measure absolute growths or declines of particular microorganisms solely with relative abundances as, for example, the increase of one taxon leads to the concurrent decrease of other(s) [17] (Fig. 1). Analysing relative abundance data using inappropriate statistical tools (such as parametric statistical tests e.g. ANOVA and measures of correlation e.g. Spearman's rank correlation) can yield up to $100 \%$ false detection rates and their application contributes to a general lack of reproducibility among microbiome studies $[18,19]$.

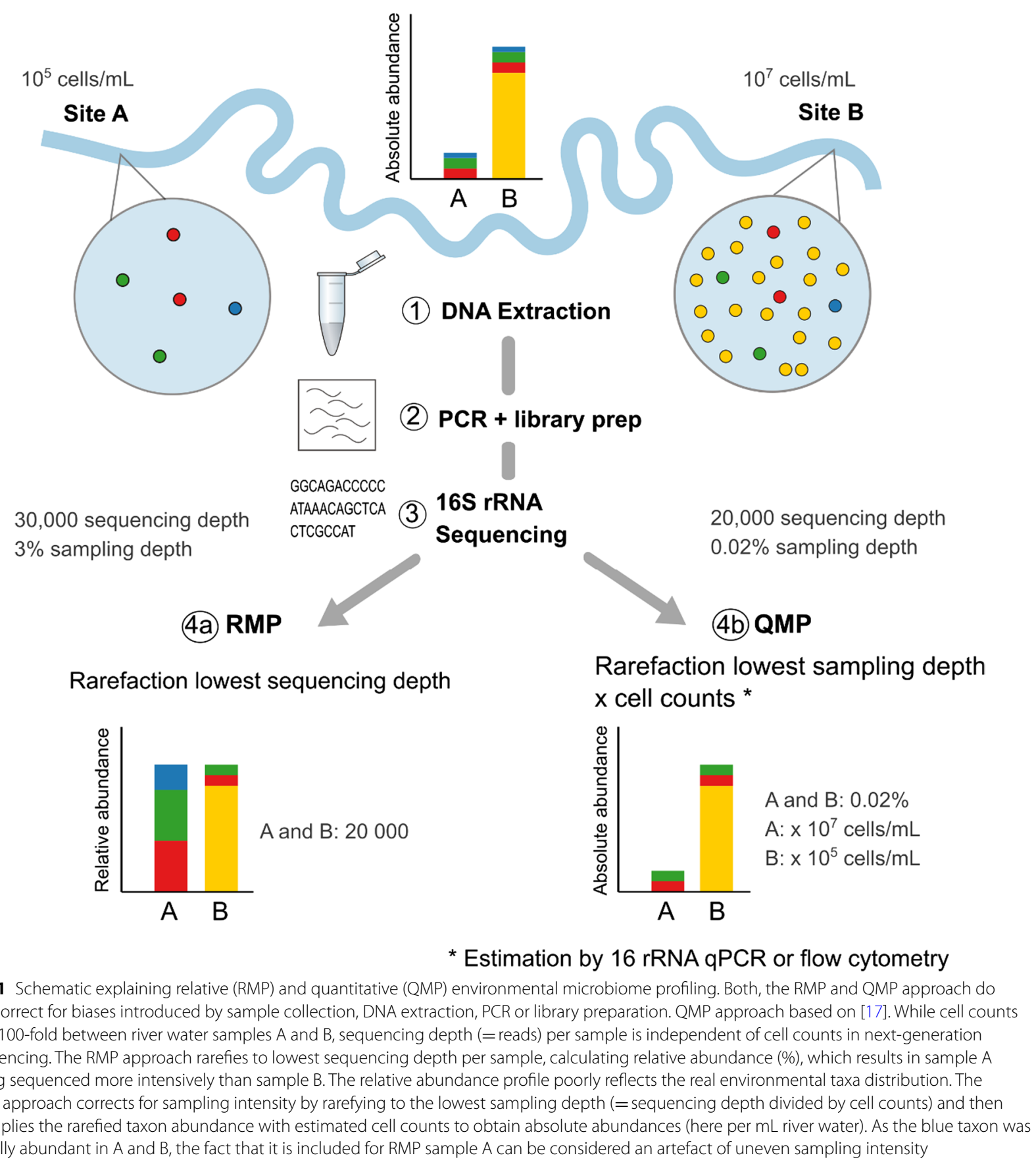


While compositional approaches are available [16], the gold standard requires cell count estimates to calculate absolute abundances [7, 19]. Such a quantitative approach can also correct sequencing data for sampling intensity to account for varied microbial loads across samples [17] (Fig. 1). Despite environmental studies routinely providing cell count estimates, these data are rarely used to calculate absolute microbial taxon abundances [20], with no studies correcting for sampling intensity [17]. Instead of relative microbiome profiling (RMP), we contend that environmental researchers should use quantitative microbiome profiling (QMP, [17]) to represent a more accurate picture of relationships between microbiomes, resistomes and metadata, guiding future QMRA applications.

The RMP approach rarefies to the lowest sequencing depth per sample, calculating relative abundance (\%). In contrast, the QMP approach as introduced by [17] corrects for sampling intensity by rarefying to the lowest sampling depth (= sequencing depth divided by cell counts) and then multiplies the rarefied taxon abundance with estimated cell counts to obtain absolute abundances (e.g. per mL surface water, Fig. 1).

Characterising and comparing anthropogenic impacts on environmental microbiomes (e.g. sewage entering rivers, waste leaching, land runoff etc.) is generally hindered by the use of varying microbial diversity indices across studies [21-23]. For a more meaningful quantification, 'diversity' needs to be unambiguously defined and applied in microbiome research [24]. Common diversity indices such as the Shannon and Simpson index do not measure diversity, but uncertainty and probability, respectively [23]. In contrast, Hill numbers (Fig. 2) provide a statistical framework that unifies and generalizes popular indices, and are intuitive and flexible enough to address a wide range of scientific questions [23, 25, 26]. Hill numbers were first proposed almost 50 years ago [26], but despite their continued appraisal [23-25], their use in microbiome research is rare [27, 28], especially for environmental microbiomes [29]. Hill numbers $\left({ }^{\mathrm{q}} \mathrm{D}\right.$, where superscript $\mathrm{q}$ describes the order of diversity) also have several additional advantages over other common diversity indices (Table 1).

Despite clear advantages in using Hill numbers [30] and the QMP approach [17] for improving reliability and comparability of environmental microbiomes, their application is rare [17, 27], and to our knowledge, has never been combined. Here we provide a workflow for combining QMP (based on parallelization of amplicon sequencing and 16S rRNA qPCR data to estimate cell counts) with absolute resistome profiling (based on high-throughput qPCR for almost 300 ARGs and

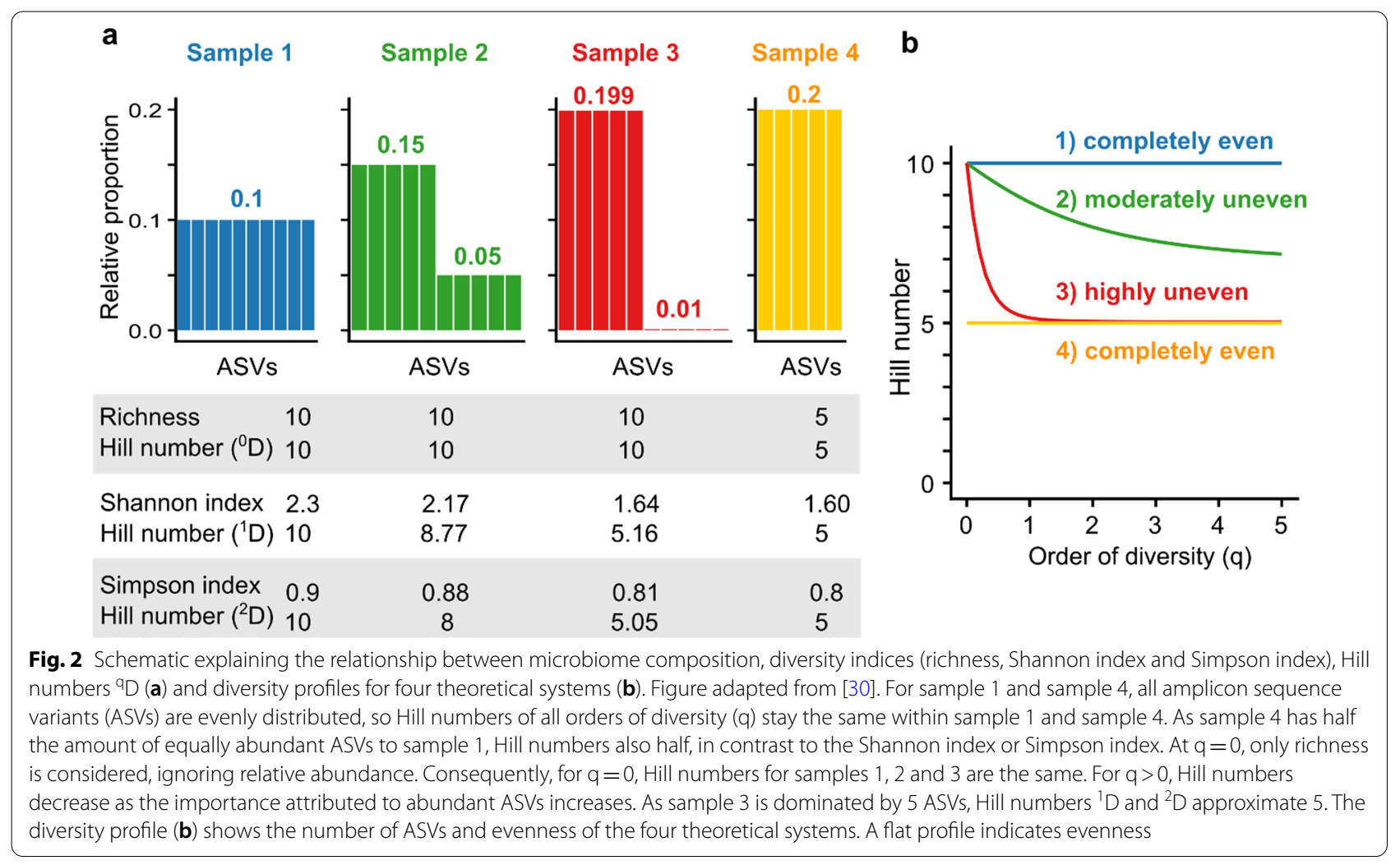


Table 1 Advantages of Hill numbers in comparison to standard diversity indices

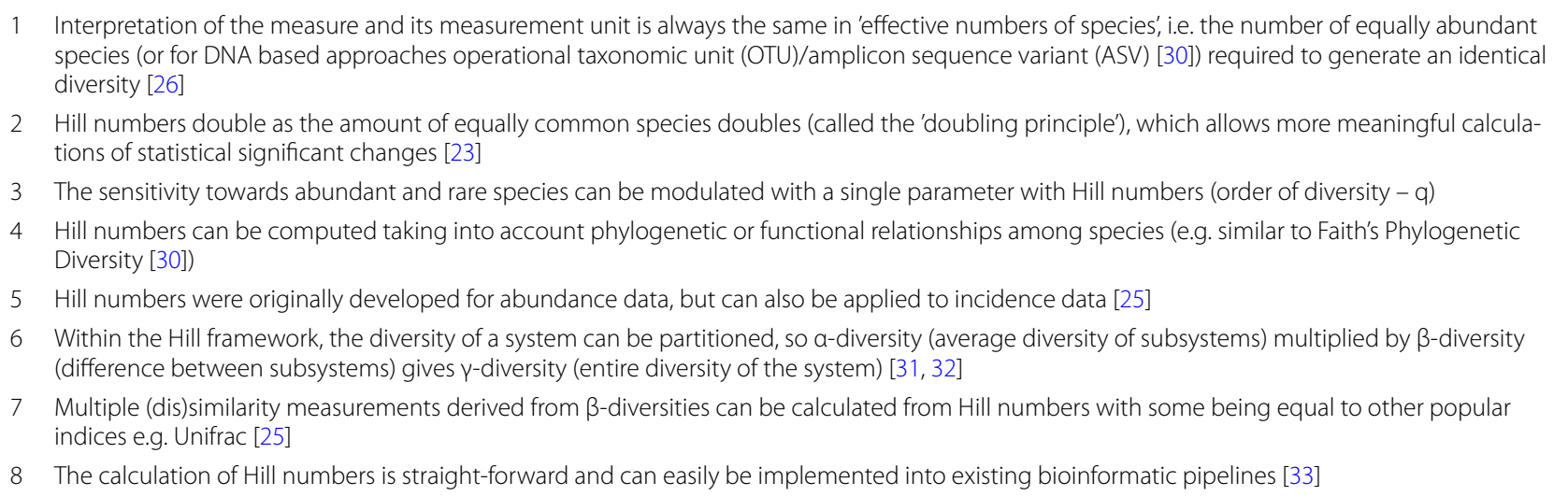

MGEs) to monitor AR in an impacted river. Such absolute microbiome profiling bypasses compositional effects in the reconstruction of microbiota interaction networks, allowing one to investigate correlations of taxa with ARGs and MGEs essential for QMRA. We also show the benefits of using the unified Hill number diversity framework to compare microbial community dynamics over space and time and confirm how misleading RMP approaches are for interpreting environmental microbiome and resistome data.

\section{Methods}

\section{Sample collection and DNA extraction}

We collected river water samples (3 technical replicates $\mathrm{x} 1 \mathrm{~L})$ from the Skudai catchment, Malaysia (288 $\mathrm{km}^{2}$, Additional file 1: Fig. S1) at eight sampling points $(6 \times$ main river and $2 \times$ tributaries $)$ during five sampling trips to capture seasonality $(1 \times$ November 2017, $2 \times$ March 2018 and $2 \times$ July 2018). The catchment is located in a humid tropical climate and is characterised to equal parts by agriculture (of that, $80 \%$ oil palm, $20 \%$ rubber plantations), forest and built-up areas [34, 35]. In total, 38 samples (with each three technical replicates) were collected with five biological replicates for the main Skudai river (S1, S2, S5, S6, S7, S8) and four biological replicates for the tributaries Melana (M5) and Senai (Se1). Some data related to this analysis was included in a previous manuscript proposing surrogate markers for predicting AR 'hot spots' in rivers where limited data are available (e.g., physico-chemical and ARG data from 30 samples [36]). Here we focus on new methods of data analysis using some of the same data but used in a different manner.

On-site, we monitored river water temperature and dissolved oxygen contents. Between $80-250 \mathrm{~mL}$ of river water was filtered onto $0.22 \mu \mathrm{m}$ cellulose-nitrate filters to extract DNA with the FastDNA SPIN kit for soil (MP Biomedicals). DNA was cleaned with the QIAquick Nucleotide Removal Kit (Qiagen). DNA quality and quantity were measured with NanoDrop and the Qubit dsDNA HS assay (both Thermo Fisher Scientific), respectively. The three technical replicates were pooled to have sufficient DNA for downstream processes. DNA was stored at $-20{ }^{\circ} \mathrm{C}$.

\section{S rRNA qPCR to estimate cell concentration}

$16 \mathrm{~S}$ rRNA qPCR assays were performed in triplicate with 16S rRNA 1055f-1392r primers [37] and SsoAdvanced Universal SYBR Green Supermix (Bio-Rad) on the BioRad CFX C1000 System (Bio-Rad) following thermocycle program: (i) 2 min of initial denaturation at $98^{\circ} \mathrm{C}$, and 40 cycles of (ii) $5 \mathrm{~s}$ denaturation and $98^{\circ} \mathrm{C}$, and (iii) $5 \mathrm{~s}$ annealing/extension at $60{ }^{\circ} \mathrm{C}$ [38]. Melt curve analysis and gels were performed. DNA samples were diluted to a working solution of $5 \mathrm{ng} / \mu \mathrm{L}$ and an internal control DNA (gfp_qPCR_f: TCGGTTATGGTGTTCAATGC; gfp_qPCR_R: GACTTCAGCACGTGTCTTGTAG) was used as inhibition controls for the qPCR. Standard curves of each set of primers were constructed using plasmid clones of the target sequences of between $10^{2}$ and $10^{8}$ copy numbers, used in parallel with each qPCR run. Cell concentration was estimated by dividing the $16 \mathrm{~S}$ rRNA concentration by 4.1 , the estimated average $16 \mathrm{~S}$ rRNA GCN per bacterium [39]. We did not incorporate individual $16 \mathrm{~S}$ GCN adjustments on the sequencing reads $[17,40]$ as current correction approaches were found to introduce rather than reduce biases [41]. The resolution of Illumina MiSeq often only allows ASV characterisation to genus level, but already within species, 16S rRNA gene copy number (16S GCN) can vary widely (e.g. 6 to 11 16S GCN for Escherichia coli [42]). 
High-throughput qPCR to quantify the resistome

High-throughput qPCR (HT-qPCR) of ARGs and MGEs was performed using SmartChip Real-Time PCR (Wafergen). A total of 296 primer sets (Additional file 1: Table S8) were used to detect 283 ARGs (52 $\beta$-lactams, 51 non-specific efflux pumps, 46 MLSBs, 39 tetracyclines, 36 aminoglycosides, 32 vancomycins, 11 others, 9 FCA, 7 sulfonamides), 12 MGEs (8 transposases, 4 integrases) and one $16 \mathrm{~S}$ rRNA gene as previously described $[43,44]$. Amplification efficiency had to be within the range of $90 \%-110 \%$ and was only confirmed when all three technical replicates were positive. Relative copy number of ARGs and MGEs were calculated and transformed to absolute copy numbers by multiplying with $16 \mathrm{~S}$ rRNA concentration for each sample. ARG and MGE cell concentrations were estimated by dividing the $16 \mathrm{~S}$ rRNA concentration by 4.1 , the estimated average $16 \mathrm{~S}$ rRNA GCN per bacterium [39].

\section{S rRNA sequencing and bioinformatics}

The hypervariable V4 region 515F-806R [45] of the $16 \mathrm{~S}$ rRNA gene was sequenced on the Illumina MiSeq platform with V2 500 cycle chemistry at NU-OMICS, Northumbria University, UK. Sample preparation and sequencing followed the Schloss MiSeq Wet Lab SOP [46] with the only deviation of spiking a 4.5 pM library, as opposed to $4 \mathrm{pM}$. Sequencing included a positive control (mock community, ZymoBIOMICS Microbial Community DNA Standard, Zymo Research), negative control (water), and extraction control (extracted water). Raw sequences were processed with QIIME2 v.2019.4 [47]. Reads were denoised into ASVs with DADA2 [48, 49], assigning ASVs to genus level with the SILVA reference database ( $\mathrm{v}$ 138) [50-52]. The V4 primer region 515F-806R was extracted from the SILVA 138 SSU NR99 dataset to retain more sequences within this region as opposed to using primer sequence to find and remove the corresponding region in the QIIME2 environment [53]. The SILVA 138 V4 classifier was trained with the machine learning software library scikit-learn v.0.20.0 using Naïve Bayes methods (fit-classifier-naive-bayes [54]) through the feature-classifier plugin [55]. The taxonomy was assigned through the same plugin, using the sklearn-based taxonomy classifier (classify-sklearn [54]). Accounting for MiSeq bleed-through between runs [56], rare ASVs of less than $0.1 \%$ of the mean sample depth were removed. The taxonomy and ASV table biom file [49] were produced for downstream analysis in $\mathrm{R}$ [57] with the phyloseq ( $\mathrm{v}$ 1.34.0) [58] and vegan ( $\mathrm{v} 2.5-7$ ) [59] package. ASVs not classified at phylum level were removed, resulting in a total of 2735 taxa for 38 samples with minimum 12,712 and maximum 83,570 reads.
Quantitative and relative microbiome profiling

For QMP, we rarefied samples to an equal sampling depth (ratio between sequencing depth and cell counts (Additional file 1: Fig. S2)) with the R function rarefy even_sampling_depth (seed 711) [17]. Reads were not corrected for individual $16 \mathrm{~S}$ rRNA GCN. The resulting rarefied abundances were multiplied with the estimated cell concentration per sample to obtain absolute microbial taxa abundance per $\mathrm{mL}$ of river water. For RMP, we rarefied sampled to an equal sequencing depth of 12,712 (seed 711), resulting in relative microbial abundances.

\section{Rank-based RMP and QMP comparisons}

We analysed ASV rank order changes between the RMP and QMP approach with the rank-biased overlap (RBO) measure and a genus co-occurrence network based on Spearman's correlation. RBO is a similarity measure on ranked lists, developed to measure the expected overlap of indefinite rankings [60]. RBO does not require every item to appear in both rankings, is not tied to a particular prefix length and its top-weightedness can be adjusted. For the latter, parameter $p$ determines the strength of the weighting to top ranks. Raising $p$ increases the depth of comparison, e.g. for $p=0.9, p=0.95$ or $p=0.97,85 \%$ of the RBO measure focus on the first ten, first 20 or first 50 results, respectively [60]. We calculated $\mathrm{RBO}$ on the most abundant 100 ASVs with $p=0.95$ to top-weigh the first 20 results in $\mathrm{R}$ with the package gespeR ( $\mathrm{v}$ 1.23.0) [61].

For the co-occurrence patterns, we first removed unclassified or ambiguously defined ASVs at genus level and then selected ASVs present in at least $85 \%$ of samples based on the QMP data (=24 ASVs). The same 24 ASVs were also selected in the RMP data. We defined and visualised taxon-taxon associations by Spearman's correlations between pairs of taxa with Benjamini-Hochberg multiple testing correction in $\mathrm{R}$ with the packages psych (v 2.1.3) [62] and corrplot (v 0.84) [63].

\section{Resistome volcano plot}

We assessed the difference in $\log _{10}$ ARG and MGE river water concentrations between up- and downstream (S1 to S8) with the Welch's t-test, applying Benjamini-Hochberg $\mathrm{P}$ adjustment to correct for multiple testing. We plotted the $\log _{10}$ fold change against statistical significance in a volcano plot with the R package EnhancedVolcano (v 1.8.0) [64].

\section{Network analysis for microbiome and resistome correlations}

We investigated microbiome and resistome co-occurrence by calculating all possible pairwise Spearman's rank correlations among bacterial orders, ARGs and MGEs present in the river water samples $(n=38)$. Only 
statistically robust correlations with Spearman's $\rho>0.8$ and Benjamini-Hochberg multiple testing corrected $P<0.01$ [65] were included in the network. Network analysis was performed in $\mathrm{R}$ with visualisation including topological property calculations in Gephi (v 0.9.2) [66].

\section{Hill diversity analysis}

Abundance-based Hill numbers and diversity profiles for RMP and QMP were calculated and plotted with the hilldiv R package (v 1.5.1) [33]. The Sørensen-type overlap dissimilarity measure for $\mathrm{q}=1$ was used to quantify the effective average proportion of nonshared ASVs in the catchment and visualised in a NMDS plot. As the Hill number ${ }^{\mathrm{q}} \mathrm{D}$ equation $[23,30]$ is not defined for $\mathrm{q}=1$, the $R$ package hilldiv calculated ${ }^{\mathrm{q}} \mathrm{D}$ for this case with $\mathrm{q}=0.99999$ (Eq. 1).

$$
{ }^{q} D=\left(\sum_{i=1}^{S} p_{i}^{q}\right)^{1 /(1-q)}
$$

${ }^{\mathrm{q}} \mathrm{D}$ Hill number, q Order of diversity, S Species richness, $\mathrm{p}_{\mathrm{i}}$ Proportional abundance of species $\mathrm{i}$.

\section{Statistical analysis and graphics}

We performed all statistical analysis in $\mathrm{R}(\mathrm{v}$ 4.0.5) [57]. We composed graphics using ggplot2 (v 3.3.3) [67] with finalisations in Inkscape ( $v$ 1.0.2) [68] except for where stated differently. The Skudai catchment map was composed in ArcGIS (v 10.6.1) [36, 69]. To assess statistically significant difference in microbiomes and resistomes between upstream (S1) and downstream (S8), we tested for normality with the Shapiro-Wilk test, followed by comparisons with the Welch's-test [70]. Effect size was measured with Cohen's D with the $\mathrm{R}$ package effsize ( $\mathrm{v}$ 0.8.1) [71].

\section{Results}

\section{Relative and absolute microbial taxa abundances}

For this study, we collected river water samples in a Malaysian rural-to-urban catchment from eight sampling points over five field trips in different seasons (total $\mathrm{n}=38$ with four to five biological replicates per site, see Additional file 1: Fig. S1). Our previous sub-study for this catchment found no large statistically significant seasonal effects for water quality and resistome data [36]. Consequently, mean concentrations with standard deviations are reported per sampling point across seasons. We estimated river water cell concentrations with 16S rRNA qPCR, correcting for multiple $16 \mathrm{~S}$ rRNA gene copies per cell. In the catchment, cell counts varied more than 100 -fold across samples with mean upstream concentrations of $(9 \pm 3) \times 10^{5}$ cells $/ \mathrm{mL}(\mathrm{S} 1)$ and mean downstream concentrations of $(2 \pm 1) \times 10^{7}$ cells $/ \mathrm{mL}$ (S8) (Additional file 1: Fig. S2).

River water microbiomes were assessed by $16 \mathrm{~S}$ rRNA sequencing with Illumina MiSeq, classifying ASVs to genus level. After data quality filtering, reads varied from 12,712 to 83,570 (median 28,187, Additional file 1: Fig. S2). Sampling depth (i.e., reads/cell count) was highest in upstream samples (S1; mean 3.4\%), with lower sampling depths obtained elsewhere in the catchment (mean 0.16-0.59\%, Additional file 1: Fig. S2). The lower cell counts upstream resulted in $\mathrm{S} 1$ samples being $21 \times$ more intensely sampled in the microbiome analysis than the most downstream site, S8 (Additional file 1: Fig. S2).

For RMP normalization, samples were rarefied to equal sequencing depth (i.e., number of reads per sample; here 12,712 reads, Additional file 1: Fig. S4). Despite known problems [72], the RMP approach remains the common practice in environmental microbiome research to calculate relative abundances of taxa (Fig. 3) [73]. For QMP [17], samples were rarefied to equal sampling depth (here $0.05 \%)$ and multiplied with the estimated cell counts per sample to obtain absolute abundance of taxa per $\mathrm{mL}$ river water (Fig. 3). In contrast to [17], individual 16S rRNA gene copy number (16S GCN) adjustment was not performed because related methods are imprecise, introducing additional bias [41].

The most abundant ASVs (based on QMP, Additional file 1: Table S1) were Cloacibacterium, Acinetobacter, C39 (genus level), and Comamonadaceae (family level). When comparing taxa changes across the catchment, the RMP barplot (Fig. 3a) might lead an inexperienced researcher to misleading conclusions. For example, the RMP barplot might be read as Comamonadaceae decreasing as one moves downstream (S1 $\rightarrow$ S8). However, when one takes cell counts into consideration (Fig. 3b), Comamonadaceae concentrations actually increase from upto downstream, which appears logical given progressive waste inputs along the river.

As relationships between microbiomes and metadata are often explored using non-parametric rank-based methods, we assessed whether the ASV rank order was conserved in the QMP vs RMP approaches. Out of the 20 most abundant ASVs determined with QMP, 16 also were present in the top 20 ASVs from the RMP approach, but only three ASVs were at the same rank order in both listings (Additional file 1: Table S1). Assessing the similarity of the rank order of the 100 most abundant ASVs with the rank-biased overlap for top-weightedness [60], we found that only $32 \%$ of the QMP and RMP results were in common (score 0.32 with $p=95$, focussing $86 \%$ of the weight on top $20 \mathrm{ASVs}$ ), suggesting the two methods providing different pictures of the system-RMP only 


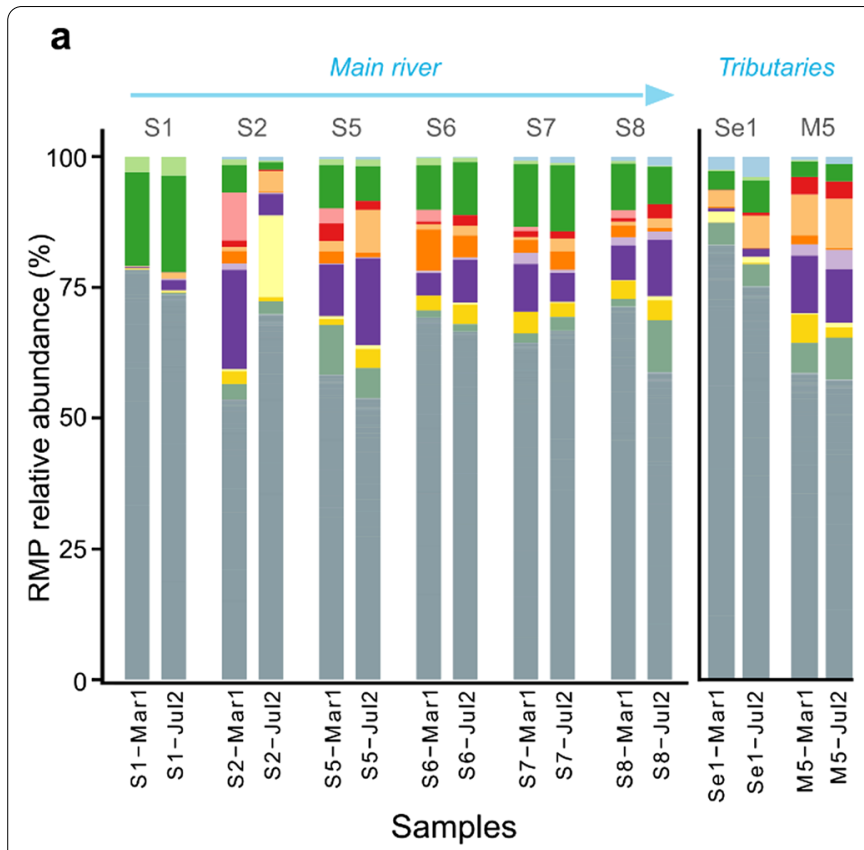

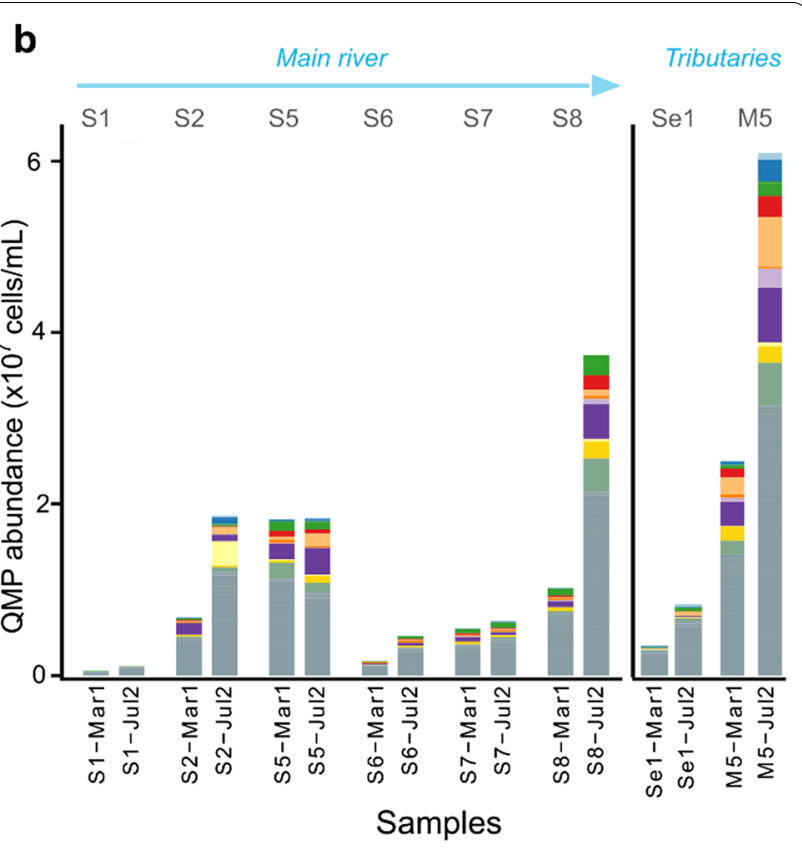

Samples

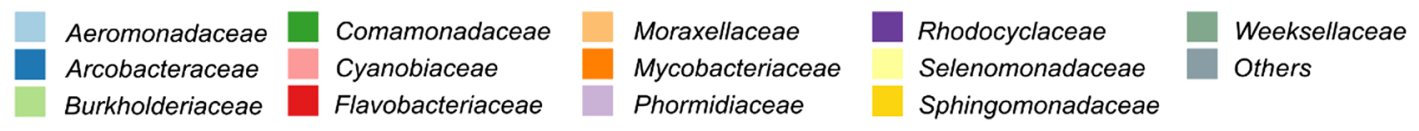

Fig. 3 Barplots showing the 20 most abundant ASVs grouped into families with remain pooled into 'Other' for the relative (RMP; a) and quantitative (QMP; b) microbiome profiling approach, analysing river water samples from eight sampling points for two sampling campaigns (March and July 2018, $n=16)$ ). See Additional file 1: Fig. S3 for all 38 samples

provides composition, whereas QMP provides composition and abundance in tandem.

Correlation analyses are often used to infer taxontaxon interactions [14]. Constructing RMP and QMP genus co-occurrence networks (Fig. 4), we detected a much larger number of significant co-varying genus pairs in the QMP than RMP network (249 versus 116). The RMP network also was dominated by negative correlations. None of the moderate to strong RMP correlations $(P<0.05$, Spearman's $\rho-0.5$ to -1$)$ were detected in the QMP correlation matrix (Fig. 4).

\section{Hill numbers for microbial diversity}

Within the Hill framework, microbial diversity can be calculated for subsystems ( $\alpha$-diversity; the sampling locations), the entire system ( $\gamma$-diversity; the river catchment), and the difference between subsystems ( $\beta$-diversity; between sampling points), all expressed using one unit, the effective number of ASVs [30]. The importance of 'richness' (ASV count in a community) and 'evenness' (equality of ASV frequency in a community) to the overall diversity can be modulated with the parameter q [74]. For diversity of order zero $(q=0)$, the Hill number is a 'richness' value because it becomes insensitive to ASV frequency, which overweighs rare ASVs. At $\mathrm{q}=1$ (exponential of Shannon index), ASVs are weighed by their frequency without favouring rare or abundant ASVs. For $\mathrm{q}=2$ (inverse of Simpson index), abundant ASVs are overweighted [23]. While specific $q$ values can be selected to calculate diversity, using $\alpha$-diversities at $q=0, q=1$ and $q=2$ together allows one to assess the degree of dominance in a community (Additional file 1: Fig. S5). This information can be summarized in a 'diversity profile,' a graph of diversity versus q, visualising the contributions of richness and evenness to a community's diversity (Fig. 5). The richer a community (higher ASV count), the higher the graph starts, whereas the more uneven the community (few dominant ASVs), the steeper the slope of the graph [23].

Microbial diversities at each sampling point in the RMP diversity profile were closely aligned, with clearer differentiation seen for the QMP data (Fig. 5a, b). Both approaches showed microbial diversity was lower upstream (S1) than elsewhere in the catchment, but spatial differences were smaller using RMP (Additional file 1: Fig. S5). This trend also was observed when calculating the Shannon and Simpson index (Additional file 1: Fig. S4). Further, $\gamma$-diversity of the catchment was 


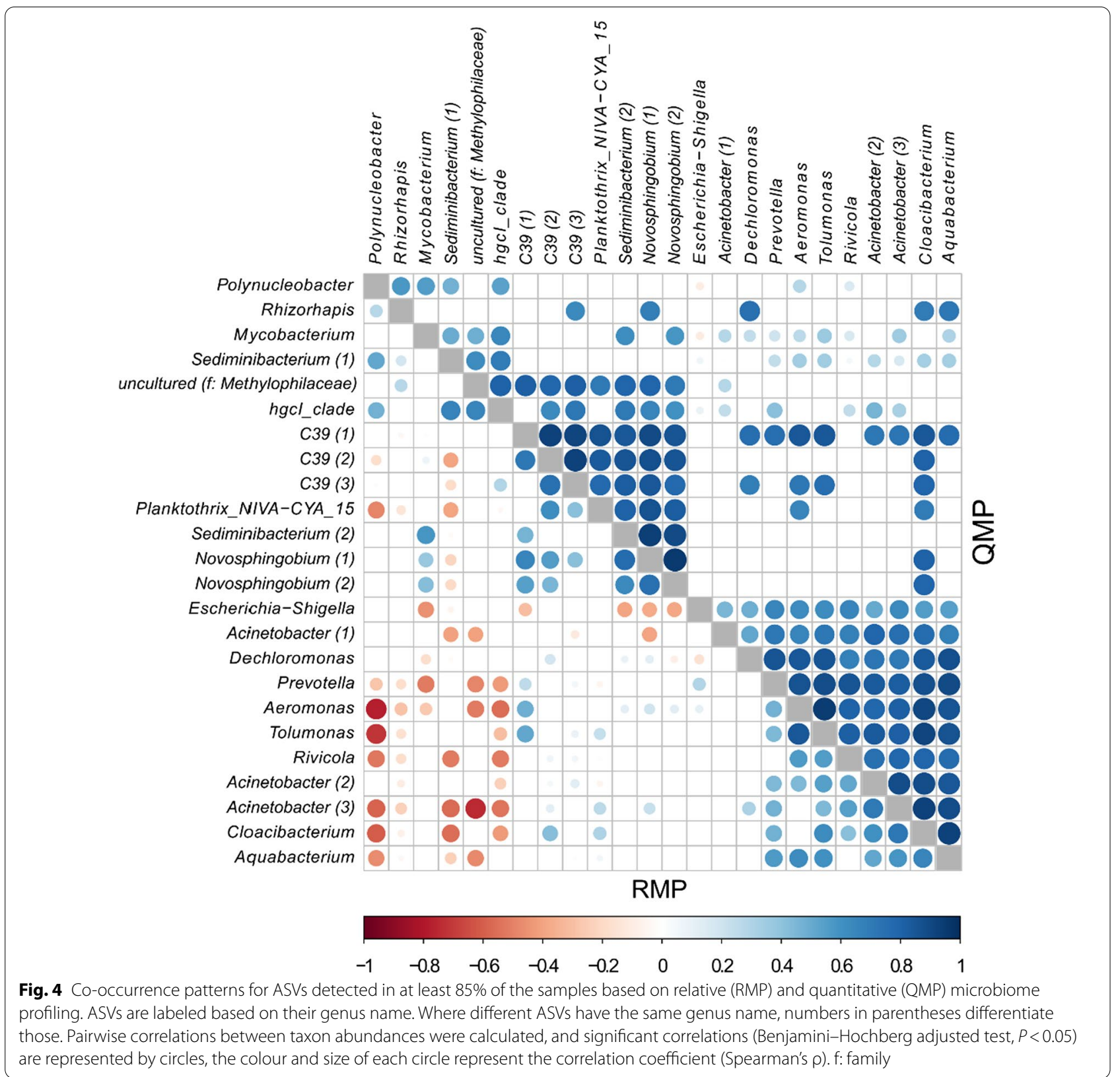

higher using the RMP versus the QMP approach, but the values for two approaches converged for $q>0$. For RMP, $\gamma$-diversity in effective numbers of ASVs was $2721(\mathrm{q}=0)$, $338(\mathrm{q}=1)$ and $96(\mathrm{q}=2)$ and for QMP, the values were $2428(\mathrm{q}=0), 328(\mathrm{q}=1)$ and $96(\mathrm{q}=2)$.

Results from the RMP and QMP approach differed most in their diversity calculations for the least impacted upstream sampling point $\mathrm{S} 1$ (mean difference $\alpha$ at $\mathrm{q}=0$ was 272 effective number of ASVs, Additional file 1: Fig. S5) with the QMP approach better correcting for varying sampling depths (Additional file 1: Fig. S2), thus avoiding 'over-sequencing. For the QMP approach
(Fig. 5b), the upstream microbial community (S1) was significantly less diverse for $\mathrm{q}=0$ and $\mathrm{q}=1$ than the farthest downstream (S8) (Welch's t-test with $\mathrm{P}<0.05$ and large Cohen's D effect size $<-0.8$, Additional file 1: Table S2). At S1, the microbial community also was more even than at any other sampling point downstream (Fig. 5b).

Comparing the $\alpha$-diversities for the tributaries Se1 and M5 (Additional file 1: Fig. S5) further shows the benefit of reporting Hill numbers at varying $q$ values. While the tributaries have similar diversities at $\mathrm{q}=0$ (richness), the diversities for $\mathrm{q}>0$ (taking frequency into account) 

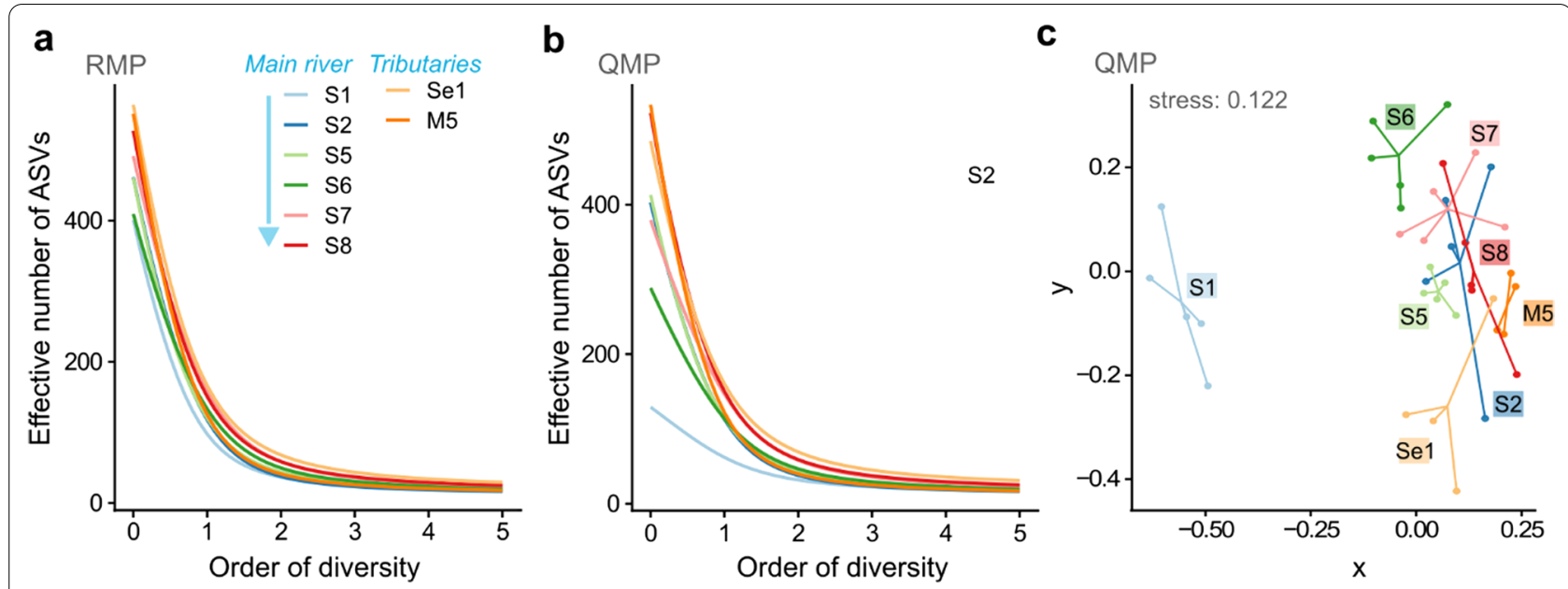

Fig. 5 Microbial diversity calculated within the Hill framework across the river catchment. Hill diversity plots represent a-diversities per sampling point based on the relative (a) and quantitative (b) microbiome profiling approach for varying q values. NMDS Sørensen-type overlap dissimilarity plot (c) is based on $\beta$-diversity, calculated for the QMP data with $q=1$. Data represented $(n=38)$ for the eight sampling points is based on five biological replicates for the main river (S1, S2, S5, S6, S7, S8) and on four biological replicates for the tributaries (Se1, M5)

decrease more rapidly for the heavily polluted M5 [36], showing a more uneven microbial community in comparison to the less polluted Se1 (Fig. 5b, Additional file 1: Fig. S5).

Within the Hill framework, dissimilarity matrices are based on $\beta$-diversities [31, 32]. We used the Sørensentype overlap dissimilarity measure for $\mathrm{q}=1$ to quantify the effective average proportion of nonshared ASVs in the catchment [33] (Fig. 5c). The NMDS plot shows the changing community structure as one moves from rural upstream (S1) to more urbanised downstream (Fig. 5c).

\section{Characterising the river resistome}

We quantified the river water resistome by applying high-throughput qPCR with 283 ARG, eight transposase and four integron primers. For this paper, we define the sum of transposase genes plus integron genes as MGEs, although we recognise that this is only an estimate based on the limited number of genes we quantified. In total, 211 ARGs ( $75 \%$ of those assayed) were detected in the river catchment with 70 ARGs $(25 \%$ of assay) shared between all river water samples $(n=38$ samples). All 12 MGEs were measured at least once in the sample with eight MGEs (75\% of assay) shared across all samples $(n=38)$ (Additional file 1: Table S3). Detected ARGs encoded resistance to eight classes of antibiotics, with $\beta$-lactam resistance being the most common (45 detected/52 in the assay) (Additional file 1: Table S3).

Summarizing ARGs and MGEs, their detected numbers (number of ARGs or MGEs), river water concentrations $\left(\log _{10}\right.$ ARG or MGE copies/mL) and cell concentrations (ARG or MGE copies/cell) all significantly increased from upstream (S1) to downstream (S8) (Welch's t-test with $P<0.05$ and large Cohen's D effect size $<-0.8$, Additional file 1: Table S4) with the Melana tributary frequently having the highest ARG and MGE concentrations (Additional file 1: Fig. S6, Additional file 1: Table S5). River water ARG concentrations increased more than two $\log _{10}$ steps along the catchment with ARG copy numbers per cell increasing from 0.1 copies/cell upstream to 2.2 copies/cell downstream (Additional file 1: Fig. S6, Additional file 1: Table S5).

The most abundant ARGs in the catchment encoded resistance against sulphonamides (sul2), aminoglycosides (aadA1, aadA2,), $\beta$-lactams (blaOXA10) and for nonspecific efflux pumps (qacEdelta1, qacH) with their mean concentrations ranging between $1 \times 10^{7}$ to $2 \times 10^{6}$ gene copies $/ \mathrm{mL}$ river water (Additional file 1: Table S6).

To assess the resistome changes along the river, we plotted ARG and MGE $\log _{10}$ fold river water concentration changes from up- to downstream (S1 to S8) against statistical significance in a volcano plot (Fig. 6). 146 ARG and MGE concentrations increased significantly at least tenfold between up- and downstream (Welch's t-test, Benjamini-Hochberg adjusted $P<0.05$ ). Four ARGs encoding for aminoglycoside, MLSB and tetracycline resistance and integron 3 increased more than four $\log _{10}$ steps from up- to downstream (Fig. 6).

\section{Network analysis of microbiomes and resistomes}

Network analysis has been proposed to explore the associations between microbiomes and resistomes, but to date, such networks have been either based on relative values [10] or semi-quantitative data (relative NGS data 


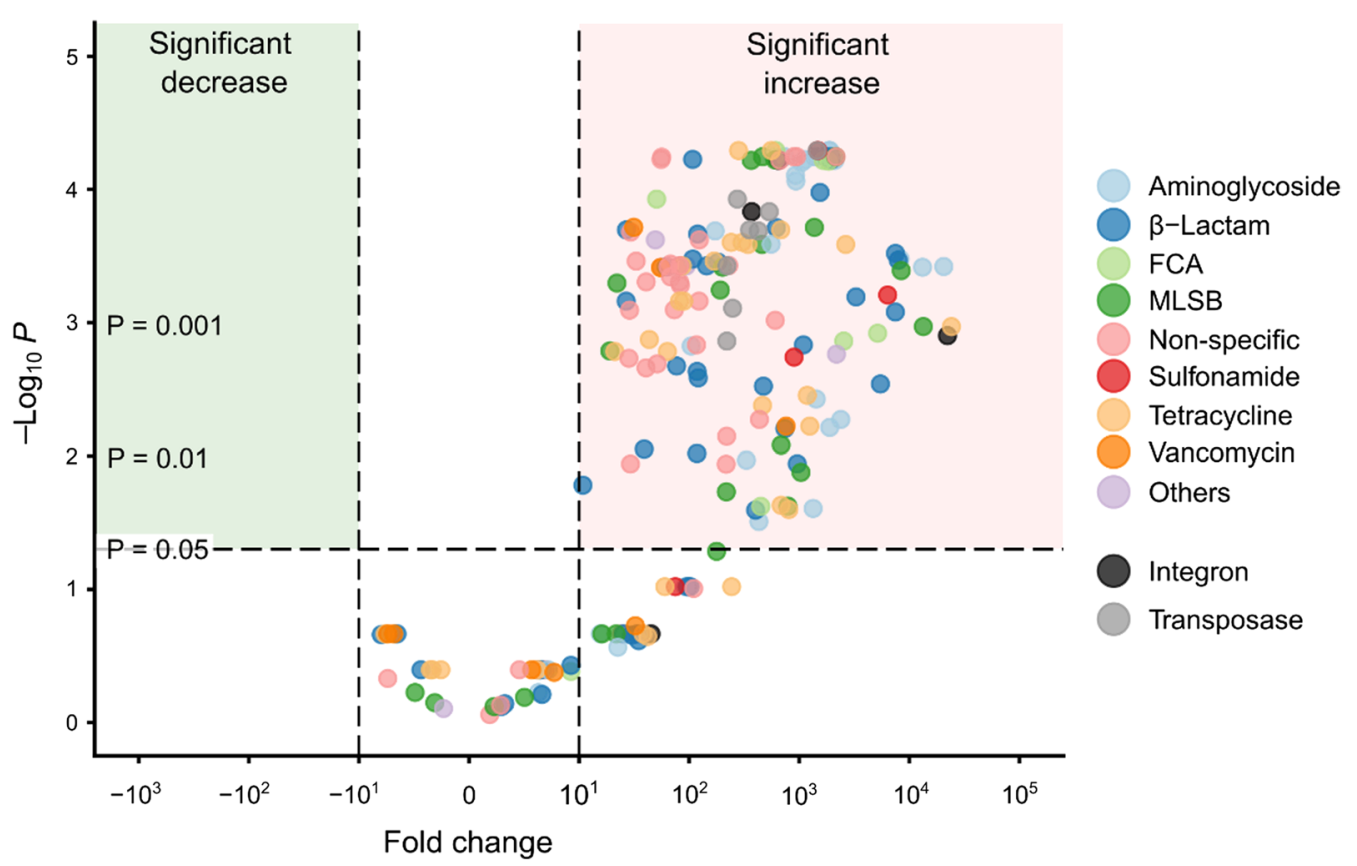

Fig. 6 Volcano plot displaying ARG and MGE $\log _{10}$ fold river water concentration changes between upstream (S1) and downstream (S8). Statistical significance calculated with the Welch's t-test, applying Benjamini-Hochberg P adjustment. Fold change calculated by subtracting mean ARG or MGE river water concentration for S1 $(n=5)$ from S8 $(n=5)$

for microbiomes and absolute HT-qPCR for resistomes [11], see Fig. 7a). Combining QMP (rather than RMP) with HT-qPCR data allows one to more fully compose the quantitative networks (Fig. 7b), overcoming negative correlation biases and spurious associations reported for relative abundance co-occurrence networks [16]. Based on the absolute taxa abundance data, the QMP network had a higher number of nodes and edges with a higher average node connectivity (=average degree) than the RMP network (Fig. 7, Additional file 1: Table S7). While for the QMP network, 36 taxa at order level had strong correlations (Spearman's $\rho>0.8$ and $P<0.01$ ) with at least three other nodes, this was only the case for 13 taxa in the RMP network (Fig. 7, Additional file 1: Table S7).

For the QMP network, the most connected ARGs, transposases, and integrons were blaOXA10 (152 degrees), tnpA 02 (147 degrees) and clinical integron 1 (clintl1; 146 degrees), respectively (Fig. 7b). The most correlating taxa belonged to the order of Burkholderiales (141 degrees), Flavobacteriales (135 degrees) and Campylobacterales (134 degrees), indicating that these bacteria might be frequent hosts of ARGs, and/or that these bacteria came from a similar source to the ARGs and MGEs (Fig. 7b). While these correlations do not replace further monitoring, they help in hypothesis formulation, addressing better-grounded research questions [14].

\section{Discussion}

Our understanding of complex environmental microbiomes has been hindered by overly relying on relative abundance data and inconsistent definitions of diversity in describing microbial changes. This hampers the ability of environmental researchers to reliably link microbiome and resistome changes in the investigation of AR fate and spread, and other practical questions [7], such as providing quantitative data for QMRAs-a crucial knowledge gap for assessing environmental AR exposure risk.

To date, few papers have reported absolute taxa abundances [20, 40, 75] and, to our knowledge, only one human study [17] used rarefaction to make sampling depths equal prior to multiplying the relative taxa abundances with cell concentrations. While this normalization step removes sequencing information for 'over-sequenced' samples (here upstream S1), it is necessary to allow a reliable comparison of microbial diversity, especially when cell counts vary widely across samples (here 100-fold). Only after sampling depth correction in QMP, did we find diversity to have increased significantly in the catchment from rural up- to urban downstream; this critical observation was not possible using the RMP approach.

Despite environmental QMP not addressing all known biases in microbiome research, it allows more accurate 


\section{Network analysis of microbiomes and resistomes}

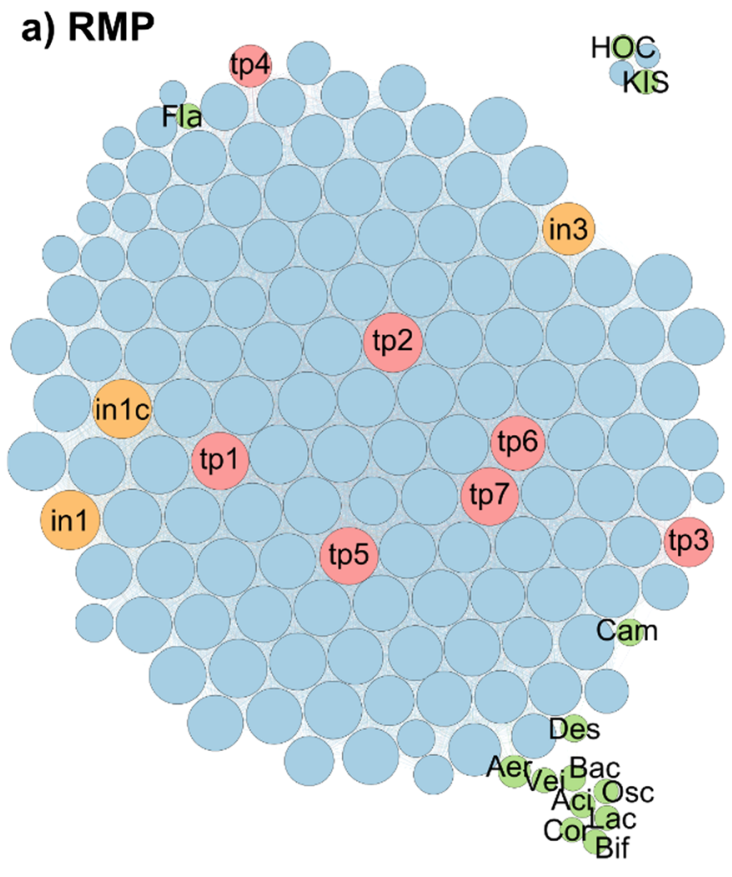

b) QMP

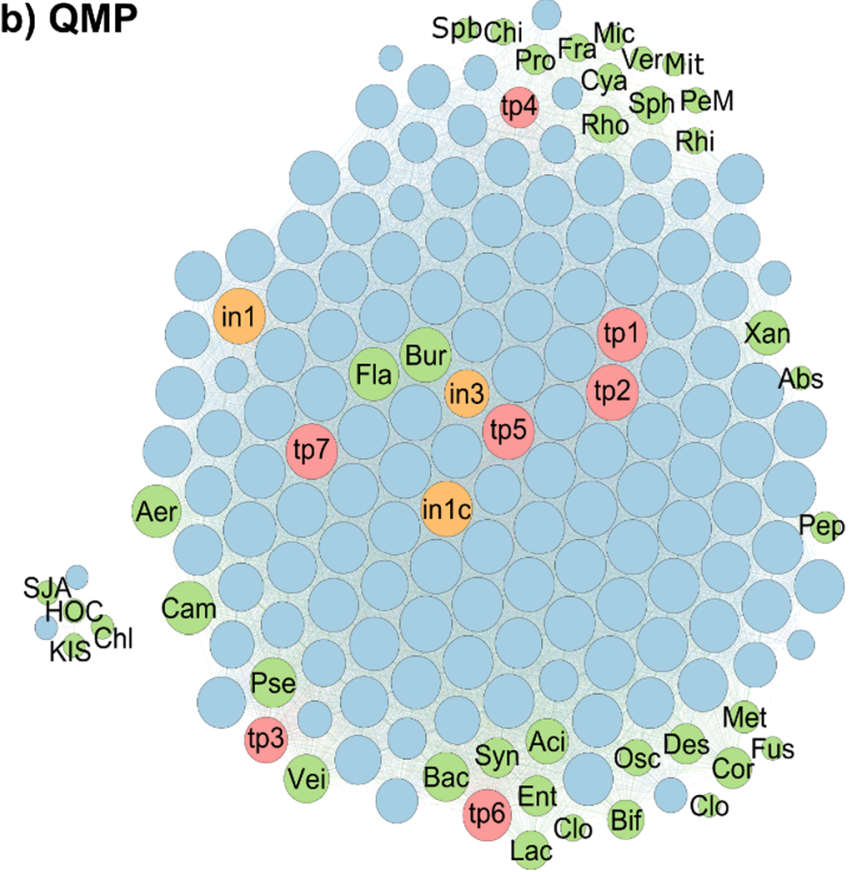

$\begin{array}{ll}\text { Order } & \text { Order } \\ \text { Abs-Absconditabacteriales SR1 } & \text { Clo- Clostridia } \\ \text { Aci-Acidaminococcales } & \text { Cor - Coriobacteriales } \\ \text { Aer - Aeromonadales } & \text { Cya - Cyanobacteriales } \\ \text { Bac- Bacteroidales } & \text { Des - Desulfovibrionales } \\ \text { Bif - Bifidobacteriales } & \text { Ent - Enterobacterales } \\ \text { Bur - Burkholderiales } & \text { Fla - Flavobacteriales } \\ \text { Cam - Campylobacterales } & \text { Fra - Frankiales } \\ \text { Chi - Chitinophagales } & \text { HOC - HOC36 } \\ \text { Chl - Chloroflexales } & \text { KIS - KIST.JJY010 }\end{array}$

\section{Order}

Lac - Lachnospirales

Met - Methanobacteriales

Mic - Micrococcales

Mit - Microtrichales

Osc - Oscillospirales

PeM - PeM15

Pep - Peptostreptococcales Tissierellales

Pro - Propionibacteriales

Pse - Pseudomonadales
Order

Rhi - Rhizobiales

Rho - Rhodobacterales

SJA - SJA 15

Sph - Sphingomonadales

$\mathrm{Spb}$ - Sphingobacteriales

Syn - Synergistales

Vei - Veillonellales Selenomonadales

Ver - Verrucomicrobiales

Xan - Xanthomonadales
Transposases

tp1 - tnpA 01

tp2 - tnpA 02

tp3 - tnpA 03

tp4 - tnpA 04

tp5 - tnpA 05

tp6 - Tp614

tp7 - tnpA 07

Integrons

in 1 - intl $1 \mathrm{LC}$

in1c - clintl1

in3 - intl3

Fig. 7 Network analysis based on relative (RMP; a) and quantitative (QMP; b) microbiome profiling, revealing co-occurrence patterns among ARGs (blue circles), MGEs (red and orange circles) and taxa at order level (green circles). A connection represents a strong (Spearman's $\rho>0.8)$ and significant ( $P<0.01$, adjusted with Benjamini Hochberg) correlation. The size of each node is proportional to the number of connections ( $=$ degree). Only nodes with at least three other connections are shown. For more details, see Additional file 1: Table S7

and easier absolute quantification of microbiota variation. In environmental studies, cell counts are routinely measured and QMP can be conducted at no extra cost, requiring little bioinformatic workflow adjustments. In this study, absolute taxa abundance data allowed to explore environmental microbiome and resistome interactions, overcoming biases related to relative taxa abundance data. Once bias is reduced, one then has more exact numerical data for QMRA calculations, which is essential for statistical and other analysis with parallel health and other end-point data within a QMRA.
Several methods are available to estimate cell counts, and one must consider the benefits and limitations of each option relative to absolute taxa abundance calculations. Here, we estimated cell counts by dividing $16 \mathrm{~S}$ rRNA qPCR concentrations with the average $16 \mathrm{~S}$ rRNA GCN per bacterium (4.1, [39]). This is a generalised approach because $16 \mathrm{~S}$ rRNA GCN can vary greatly across cells. Measuring total cells using flow cytometry is another possible option $[17,20]$. Flow cytometry protocols are available for almost all environmental compartments (e.g. wastewater [76], biofilms [77] or seawater [78]) to optimise cell detection. 
Conversely, parallel qPCR quantification of the same products as NGS is an option that might reduce bias from non-genetic methods (e.g., flow cytometry of cells vs. qPCR of $16 \mathrm{~S}$ rRNA). Recent advances now also allow the quantification of viable cells with digital PCR $[79,80]$. When using $\mathrm{qPCR}$, the same primer region should be targeted to estimate cell counts and assess the microbiome. Further qPCR bias could be reduced by diluting sample DNA instead of normalising to a DNA concentration. We recommend further research to compare cell concentration measurements for QMP.

Diversity has been defined in so many different ways that its ability to transfer accurate information on microbial community changes, e.g. due to human impact, is compromised [24]. Jost and Chao (2020) introduced the analogy that diversity indices (e.g. Shannon or Simpson index) are connected to diversity in the same manner as a sphere's diameter is connected to its volume. While the diameter is an index of the sphere's volume, it is not the volume itself. They state that using the diameter instead of volume in engineering calculations would result in chaos, but this is what biologists are currently doing with diversity indices [74]. Shannon and Simpson index are useful diversity indices with an important role in ecology, but their values provide information on uncertainty and probability, respectively, rather than measuring diversity [23]. The Hill number framework provides a better and more unified approach to calculate and compare microbial diversities across environmental compartments, especially where the parameter q can be used to modulate the sensitivity towards abundant versus rare ASVs.

Depending on the study purpose, scientists might choose to calculate Hill numbers for several q for an indepth diversity analysis (as performed here) or for one $q$ value only. To define a core microbiome or when rare ASVs are considered untrustworthy due to technical bias (e.g. PCR or sequencing errors), $q=2$ could be chosen to put more weight on abundant ASVs and results could be interpreted as effective number of dominant ASVs in the system [25, 30]. In contrast, when the rarest ASVs are as important as the most abundant ASVs, for example for conservation purposes, $q=0$ could be chosen [30]. The recently published $\mathrm{R}$ hilldiv package [33] enables DNAbased diversity calculations with Hill numbers.

In this study, we observed an increase in diversity and decrease in evenness along the river from a less polluted upstream to a more polluted downstream. Environmental $\mathrm{AR}$ increased along the river as indicated by the enrichment of ARGs and MGEs. Resistome concentrations in the heavily urbanized Melana tributary were often higher than in the river itself. The increase in diversity, together with the increasing levels of cell counts, ARGs and MGEs in this rural-to-urban catchment are likely caused by insufficiently treated sewage entering the river (as previously shown in our sub-study of the same catchment [36] and in a different study covering the same area [81]). The most abundant ASVs for this catchment were Cloacibacterium, Acinetobacter, C39 (genus level) and Comamonadaceae (family level), also common in wastewater-impacted water bodies in China and India [82-84]. Comparing co-occurrence networks of absolute taxa with absolute ARG and MGE data allowed proposing hypothesis of possible taxa harbouring AR to be further investigated in experimental studies.

\section{Conclusions}

This study shows the straightforward and easy implementation of a quantitative microbial profiling approach and intuitive diversity characterisation with Hill numbers. We recommend our new combined approach to become the norm for future environmental microbiome (and resistome) research, especially to underpin improved QMRAs. Only when such methods are employed will environmental AR studies become more quantitative and truly comparable.

\section{Supplementary Information}

The online version contains supplementary material available at https://doi. org/10.1186/s40793-021-00391-0.

Additional file 1. Supplementary information.

\section{Acknowledgements}

We acknowledge the support from research staff at NUMed, including Jia Yee Ho, Sylvia Sue Xian Liew, Mardhiah Zainal Abidin and Michaela L Goodson. We also thank colleagues from the Chinese Academy of Science for performing the HT-qPCR assay, including Xinyuan Zhou, Jian-Qiang Su and Yong-Guan Zhu.

\section{Authors' contributions}

AO performed the field work, analysed the samples, performed statistical analysis and data visualisation, and wrote the manuscript. MQB assisted in the $16 \mathrm{~S}$ qPCR analysis and provided guidance in planning the study. AMZ provided help with bioinformatics. MRMH provided local background data and knowledge. AO and DWG designed the study plan, where GO and DWG supervised the work and assisted in conceptualising the ideas and methodologies. All authors provided suggestions and reviewed the manuscript. All authors read and approved the final manuscript.

\section{Funding}

This work was funded by the Newcastle University SAgE Singapore Scholarships programme, a British Council Newton Fund Institutional Links grant (331945729), and a UK EPSRC Impact Acceleration Award (EP/K503885/1). HTqPCR analysis was funded by the Key Collaborative Research Program of the Alliance of International Science Organizations (ANSO-CR-KP-2020-03).

\section{Availability of data and materials}

Raw amplicon sequencing data that support the findings of this study have been deposited in European Nucleotide Archive with study accession number PRJEB42314. All other data can be accessed through the Center for Open Science, OSF (Ott, Amelie. 2021. 'Monitoring and Modelling of Antibiotic Resistance in Southeast Asian Rivers.'OSF. https://osf.io/gcpky/?view_only= 90e614c2c6b64483aa503694af113789). 


\section{Declarations}

Ethics approval and consent to participate

Not applicable.

\section{Consent for publication}

Not applicable.

\section{Competing interests}

The authors declare that they have no competing interests.

\section{Author details}

${ }^{1}$ School of Engineering, Newcastle University, Cassie Building, Newcastle upon Tyne NE1 7RU, UK. ² Universiti Teknologi Malaysia, Jalan Iman, 81310 Skudai, Johor, Malaysia.

Received: 9 August 2021 Accepted: 4 November 2021

Published online: 18 November 2021

\section{References}

1. WHO. Antimicrobial resistance: global report on surveillance. 2014. https://www.who.int/drugresistance/documents/surveillancereport/en/.

2. O'Neill J. Tackling drug-resistant infections globally: final report and recommendations. 2016. https://wellcomecollection.org/works/thvwsuba.

3. Hendriksen RS, Munk P, Njage P, van Bunnik B, McNally L, Lukjancenko O, et al. Global monitoring of antimicrobial resistance based on metagenomics analyses of urban sewage. Nat Commun. 2019;10:1124.

4. Hassoun-Kheir N, Stabholtz Y, Kreft J-U, de la Cruz R, Romalde JL, Nesme J, et al. Comparison of antibiotic-resistant bacteria and antibiotic resistance genes abundance in hospital and community wastewater: a systematic review. Sci Total Environ. 2020;743: 140804. https://doi.org/10.1016/j.scito tenv.2020.140804

5. Graham DW, Collignon P, Davies J, Larsson DGJ, Snape J. Underappreciated role of regionally poor water quality on globally increasing antibiotic resistance. Environ Sci Technol. 2014;48:11746-7. https://doi.org/10.1021/ es504206x

6. Huijbers PMC, Flach C-F, Larsson DGJ. A conceptual framework for the environmental surveillance of antibiotics and antibiotic resistance. Environ Int. 2019;130:104880. https://doi.org/10.1016/j.envint.2019.05.074.

7. Rice EW, Wang P, Smith AL, Stadler LB. Determining hosts of antibiotic resistance genes: a review of methodological advances. Environ Sci Technol Lett. 2020;7:282-91.

8. Hultman J, Tamminen M, Pärnänen K, Cairns J, Karkman A, Virta M. Host range of antibiotic resistance genes in wastewater treatment plant influent and effluent. FEMS Microbiol Ecol. 2018;94:1-10.

9. Belton JM, McCord RP, Gibcus JH, Naumova N, Zhan Y, Dekker J. Hi-C: a comprehensive technique to capture the conformation of genomes. Methods. 2012:58:268-76. https://doi.org/10.1016/j.ymeth.2012.05.001.

10. Li B, Yang Y, Ma L, Ju F, Guo F, Tiedje JM, et al. Metagenomic and network analysis reveal wide distribution and co-occurrence of environmental antibiotic resistance genes. ISME J. 2015;9:2490-502.

11. Peng F, Guo Y, Isabwe A, Chen H, Wang Y, Zhang Y, et al. Urbanization drives riverine bacterial antibiotic resistome more than taxonomic community at watershed scale. Environ Int. 2020;137:105524. https://doi.org/ 10.1016/j.envint.2020.105524.

12. Amarasiri M, Sano D, Suzuki S. Understanding human health risks caused by antibiotic resistant bacteria (ARB) and antibiotic resistance genes (ARG) in water environments: current knowledge and questions to be answered. Crit Rev Environ Sci Technol. 2020;50:2016-59. https://doi.org/ 10.1080/10643389.2019.1692611.

13. Ashbolt NJ, Amézquita A, Backhaus T, Borriello P, Brandt KK. Review human health risk assessment (HHRA) for environmental development and transfer of antibiotic resistance. Environ Health Perspect. 2013:121:993-1001.

14. Carr A, Diener C, Baliga NS, Gibbons SM. Use and abuse of correlation analyses in microbial ecology. ISME J. 2019;13:2647-55. https://doi.org/ 10.1038/s41396-019-0459-z.
15. Debelius J, Song SJ, Vazquez-Baeza Y, Xu ZZ, Gonzalez A, Knight R. Tiny microbes, enormous impacts: what matters in gut microbiome studies? Genome Biol. 2016:17:1-12. https://doi.org/10.1186/s13059-016-1086-x

16. Gloor G, Macklaim JM, Pawlowsky-Glahn V, Egozcue JJ. Microbiome datasets are compositional: and this is not optional. Front Microbiol. 2017:8:1-6

17. Vandeputte D, Kathagen G, D'Hoe K, Vieira-Silva S, Valles-Colomer M, Sabino J, et al. Quantitative microbiome profiling links gut community variation to microbial load. Nature. 2017:551:507-11. https://doi.org/10. 1038/nature24460

18. Knight R, Vrbanac A, Taylor BC, Aksenov A, Callewaert C, Debelius J, et al. Best practices for analysing microbiomes. Nat Rev Microbiol. 2018;16:113. https://doi.org/10.1038/s41579-018-0029-9.

19. Morton JT, Marotz C, Washburne A, Silverman J, Zaramela LS, Edlund A, et al. Establishing microbial composition measurement standards with reference frames. Nat Commun. 2019;10:2719. https://doi.org/10.1038/ s41467-019-10656-5.

20. Props R, Kerckhof FM, Rubbens P, De VJ, Sanabria EH, Waegeman W, et al. Absolute quantification of microbial taxon abundances. ISME J. 2017:11:584-7. https://doi.org/10.1038/ismej.2016.117.

21. Köchling T, Sanz JL, Galdino L, Florencio L, Kato MT. Impact of pollution on the microbial diversity of a tropical river in an urbanized region of Northeastern Brazil. Int Microbiol. 2017;20:11-24.

22. Li Y, Fan L, Zhang W, Zhu X, Lei M, Niu L. How did the bacterial community respond to the level of urbanization along the Yangtze River? Environ Sci Process Impacts. 2020;22:161-72

23. Jost L. Entropy and diversity. Oikos. 2006:113:363-75.

24. Tuomisto H. Commentary: do we have a consistent terminology for species diversity? Yes, if we choose to use it. Oecologia. 2011;167:903-11.

25. Chao A, Chiu C-H, Jost L. Unifying species diversity, phylogenetic diversity, functional diversity, and related similarity and differentiation measures through hill numbers. Annu Rev Ecol Evol Syst. 2014;45:297-324.

26. Hill MO. Diversity and evenness: a unifying notation and its consequences. Ecology. 1973;54:427-32. https://doi.org/10.2307/1934352.

27. Props R, Monsieurs P, Mysara M, Clement L, Boon N. Measuring the biodiversity of microbial communities by flow cytometry. Methods Ecol Evol. 2016;7:1376-85.

28. Alberdi A, Razgour O, Aizpurua O, Novella-Fernandez R, Aihartza J, Budinski l, et al. DNA metabarcoding and spatial modelling link diet diversification with distribution homogeneity in European bats. Nat Commun. 2020;11:1-8.

29. Quintela-Baluja M, Frigon D, Abouelnaga M, Jobling K, Romalde JL, Gomez ML, et al. Dynamics of integron structures across a wastewater network-implications to resistance gene transfer. Water Res. 2021. https://doi.org/10.1016/j.watres.2021.117720.

30. Alberdi A, Gilbert MTP. A guide to the application of Hill numbers to DNAbased diversity analyses. Mol Ecol Resour. 2019;19:804-17.

31. Chao A, Chiu $\mathrm{CH}$, Hsieh TC, Inouye BD. Proposing a resolution to debates on diversity partitioning. Ecology. 2012;93:2037-51.

32. Jost L. Partitioning diversity into independent alpha and beta components. Ecology. 2007:88:2427-39.

33. Alberdi A, Gilbert MTP. hilldiv: an R package for the integral analysis of diversity based on Hill numbers. bioRxiv. 2019. https://doi.org/10.1101/ 545665.

34. Bello AD, Haniffah MRM, Hanapi MN, Usman AB. Identification of critical source areas under present and projected land use for effective management of diffuse pollutants in an urbanized watershed. Int J River Basin Manag. 2018. https://doi.org/10.1080/15715124.2018.1461108.

35. Bello AD. Modelling the impacts of land-use and climate change in Skudai river watershed. UTM; 2018. http://hdl.handle.net/2381/20324.

36. Ott A, O'Donnell G, Tran NH, Haniffah MRBM, Su J-Q, Zealand AM, et al. Developing surrogate markers for predicting antibiotic resistance "hot spots" in rivers where limited data are available. Environ Sci Technol. 2021:55:7466-78.

37. Harms G, Layton AC, Dionisi HM, Gregory IR, Garrett VM, Hawkins SA, et al. Real-time PCR quantification of nitrifying bacteria in a municipal wastewater treatment plant. Environ Sci Technol. 2003;37:343-51.

38. Quintela-Baluja M, Abouelnaga M, Romalde J, Su J-Q, Yu Y, GomezLopez M, et al. Spatial ecology of a wastewater network defines the antibiotic resistance genes in downstream receiving waters. Water Res. 2019;162:347-57. https://doi.org/10.1016/j.watres.2019.06.075. 
39. Klappenbach JA. rrndb: the Ribosomal RNA Operon Copy Number Database. Nucleic Acids Res. 2001;29:181-4.

40. Jian C, Luukkonen P, Yki-Järvinen H, Salonen A, Korpela K. Quantitative PCR provides a simple and accessible method for quantitative microbiota profiling. PLoS ONE. 2020;15:1-10.

41. Louca S, Doebeli M, Parfrey LW. Correcting for 165 rRNA gene copy numbers in microbiome surveys remains an unsolved problem. Microbiome. 2018;6:1-12.

42. Stoddard SF, Smith BJ, Hein R, Roller BRK, Schmidt TM. rrnDB: improved tools for interpreting rRNA gene abundance in bacteria and archaea and a new foundation for future development. Nucleic Acids Res. 2014;43:D593-8. https://doi.org/10.1093/nar/gku1201.

43. Zhu YG, Zhao Y, Li B, Huang CL, Zhang SY, Yu S, et al. Continental-scale pollution of estuaries with antibiotic resistance genes. Nat Microbiol. 2017;2:16270.

44. Zhu YG, Johnson TA, Su JQ, Qiao M, Guo GX, Stedtfeld RD, et al. Diverse and abundant antibiotic resistance genes in Chinese swine farms. Proc Natl Acad Sci U S A. 2013:110:3435-40.

45. Caporaso JG, Lauber CL, Walters WA, Berg-Lyons D, Lozupone CA, Turnbaugh PJ, et al. Global patterns of $16 \mathrm{~S}$ rRNA diversity at a depth of millions of sequences per sample. Proc Natl Acad Sci U S A. 2011;108(SUPPL. 1):4516-22.

46. Kozich JJ, Westcott SL, Baxter NT, Highlander SK, Schloss PD. Development of a dual-index sequencing strategy and curation pipeline for analyzing amplicon sequence data on the miseq illumina sequencing platform. Appl Environ Microbiol. 2013;79:5112-20.

47. Bolyen E, Rideout JR, Dillon MR, Bokulich NA, Abnet CC, Al-Ghalith GA, et al. Reproducible, interactive, scalable and extensible microbiome data science using QIIME 2. Nat Biotechnol. 2019;37:852-7. https://doi. org/10.1038/s41587-019-0209-9.

48. Callahan BJ, McMUrdie PJ, Rosen MJ, Han AW, Johnson AJA, Holmes SP. DADA2: high resolution sample inference from Illumina amplican data. Nat Methods. 2016;13:1-6.

49. McDonald D, Clemente JC, Kuczynski J, Rideout JR, Stombaugh J, Wendel $\mathrm{D}$, et al. The Biological Observation Matrix (BIOM) format or: how I learned to stop worrying and love the ome-ome. Gigascience. 2012;1:7.

50. Quast C, Pruesse E, Yilmaz P, Gerken J, Schweer T, Yarza P, et al. The SILVA ribosomal RNA gene database project: Improved data processing and web-based tools. Nucleic Acids Res. 2013;41:590-6.

51. Yilmaz P, Parfrey L, Yarza P, Gerken J, Pruesse E, Quast C, et al. The SILVA and "all-species living tree project (LTP)" taxonomic frameworks. Nucleic Acids Res. 2014;42:643-8.

52. McKinney W. Data structures for statistical computing in Python. In: van der Walt S, Millman J, editors. Proceedings of the 9th Python in Science Conference. 2010. p. 51-6.

53. Robeson MS. make_SILVA_db. 2020. https://github.com/mikerobeson/ make_SILVA_db. Accessed 21 May 2020.

54. Pedregosa F, Varoquaux G, Gramfort A, Michel V, Thirion B, Grisel O, et al. Scikit-learn: machine learning in Python. J Mach Learn Res. 2012;12:2825-30.

55. Bokulich NA, Kaehler BD, Rideout JR, Dillon M, Bolyen E, Knight R, et al. Optimizing taxonomic classification of marker-gene amplicon sequences with QIIME 2's q2-feature-classifier plugin. Microbiome. 2018;6:1-17. https://doi.org/10.1186/s40168-018-0470-z.

56. Loman NJ, Misra RV, Dallman TJ, Constantinidou C, Gharbia SE, Wain J, et al. Performance comparison of benchtop high-throughput sequencing platforms. Nat Biotechnol. 2012;30:434-9.

57. R Core Team. R: a language and environment for statistical computing. 2020.

58. McMurdie PJ, Holmes S. phyloseq: an R package for reproducible interactive analysis and graphics of microbiome census data. PLOS ONE. 2013;8: e61217. https://doi.org/10.1371/journal.pone.0061217.

59. Oksanen J, Blanchet FG, Friendly M, Kindt R, Legendre P, McGlinn D, et al. vegan: Community Ecology Package. R package version 2.5-6. Cran R. 2019

60. Webber W, Moffat A, Zobel J. A similarity measure for indefinite rankings. ACM Trans Inf Syst. 2010;28:1-34.

61. Schmich F. gespeR: Gene-Specific Phenotype EstimatoR. R package version 1.20.0. 2020:1-7. http://www.cbg.ethz.ch/software/gespeR.
62. Revelle W. psych: procedures for personality and psychological research. 2019. https://cran.r-project.org/package $=$ psych.

63. Wei T, Simko V. R package "corrplot": Visualization of a Correlation Matrix. 2017. https://github.com/taiyun/corrplot.

64. Blighe K, Rana S, Lewis M. EnhancedVolcano: publication-ready volcano plots with enhanced colouring and labeling. 2020. https://github. $\mathrm{com} /$ kevinblighe/EnhancedVolcano.

65. Junker B, Schreiber F. Analysis of biological networks. New Jersey: Wiley; 2008.

66. Bastian M, Heymann S, Jacomy M. Gephi: an open source software for exploring and manipulating networks. 2009. http://www.aaai.org/ocs/ index.php/ICWSM/09/paper/view/154.

67. Wickham H. ggplot2: Elegant Graphics for Data Analysis. 2016. https:// ggplot2.tidyverse.org.

68. Inkscape Project. Inkscape. 2017. https://inkscape.org.

69. ESRI. ArcGIS Desktop. 2018.

70. Delacre M, Lakens D, Leys C. Why psychologists should by default use welch's t-Test instead of student's t-Test. Int Rev Soc Psychol. 2017;30:92-101.

71. Torchiano M. effsize: efficient effect size computation. 2020. https:// cran.r-project.org/package $=$ effsize.

72. McMurdie PJ, Holmes S. Waste not, want not: why rarefying microbiome data is inadmissible. PLoS Comput Biol. 2014;10:e1003531.

73. Weiss S, Xu ZZ, Peddada S, Amir A, Bittinger K, Gonzalez A, et al. Normalization and microbial differential abundance strategies depend upon data characteristics. Microbiome. 2017;5:1-18.

74. Jost L, Chao A. Diversity analysis. Taylor \& Francis; 2020. http://www. loujost.com/Statistics\%20and\%20Physics/Diversity\%20and\%20Similari ty/SampleChapter.pdf.

75. Guo X, Zhang X, Qin Y, Liu Y-X, Zhang J, Zhang N, et al. Host-associated quantitative abundance profiling reveals the microbial load variation of root microbiome. Plant Commun. 2020;1:100003.

76. Brown MRR, Hands CLL, Coello-Garcia T, Sani BSS, Ott AIGIG, Smith SJJ, et al. A flow cytometry method for bacterial quantification and biomass estimates in activated sludge. J Microbiol Methods. 2019;160:7383. https://doi.org/10.1016/j.mimet.2019.03.022.

77. Vignola M, Werner D, Hammes F, King LC, Davenport RJ. Flow-cytometric quantification of microbial cells on sand from water biofilters. Water Res. 2018;143:66-76.

78. Ott A, Martin TJ, Acharya K, Lyon DY, Robinson N, Rowles B, et al. Multi-laboratory validation of a new marine biodegradation screening test for chemical persistence assessment. Environ Sci Technol. 2020;54:4210-20

79. Dong L, Wang S, Fu B, Wang J. Evaluation of droplet digital PCR and next generation sequencing for characterizing DNA reference material for KRAS mutation detection. Sci Rep. 2018;8:9650.

80. Santander RD, Meredith CL, Aćimović SG. Development of a viability digital PCR protocol for the selective detection and quantification of live Erwinia amylovora cells in cankers. Sci Rep. 2019;9:11530.

81. Ho JY, Jong M-C, Acharya K, Liew SSX, Smith DR, Noor ZZ, et al. Multidrug-resistant bacteria and microbial communities in a river estuary with fragmented suburban waste management. J Hazard Mater. 2020;2020(405): 124687. https://doi.org/10.1016/j.jhazmat.2020. 124687.

82. Zhou ZC, Zheng J, Wei YY, Chen T, Dahlgren RA, Shang X, et al. Antibiotic resistance genes in an urban river as impacted by bacterial community and physicochemical parameters. Environ Sci Pollut Res. 2017;24:23753-62

83. Marathe NP, Pal C, Gaikwad SS, Jonsson V, Kristiansson E, Larsson DGJ. Untreated urban waste contaminates Indian river sediments with resistance genes to last resort antibiotics. Water Res. 2017;124:388-97. https://doi.org/10.1016/j.watres.2017.07.060.

84. Chen QL, Li H, Zhou XY, Zhao Y, Su JQ, Zhang X, et al. An underappreciated hotspot of antibiotic resistance: the groundwater near the municipal solid waste landfill. Sci Total Environ. 2017;609:966-73.

\section{Publisher's Note}

Springer Nature remains neutral with regard to jurisdictional claims in published maps and institutional affiliations. 